\title{
INVESTIGACIONES
}

\section{Reflexiones de profesores en un escenario de Estudio de Clases para el desarrollo profesional}

\author{
Teachers' Reflections on Lesson Study for professional development
}

\author{
M. Soledad Montoya González \\ Universidad Alberto Hurtado, Santiago, Chile. \\ Correo electrónico: mmontoya@uahurtado.cl
}

\begin{abstract}
RESUMEN
Este artículo expone sobre una investigación centrada en analizar las reflexiones de un grupo de trabajo de profesores que pertenecían a un taller de reflexión de la práctica, enmarcado en un programa de postítulo para el desarrollo profesional y que desarrollaban la metodología de Estudio de Clases. Para ello se utilizó un enfoque cualitativo y corresponde a un estudio de casos. Se fundamentó en el constructo teórico denominado reproducibilidad y en la conceptualización reflexión y tipos de reflexión. Los hallazgos mostraron que en forma natural emergen reflexiones de tipo pedagógica y didáctica en las discusiones entre los profesores y que las reflexiones de tipo matemática son más escasas. Sin embargo, en el momento de instalar la reproducción del diseño de clase se focalizan en reflexiones de tipo matemática y didáctica.
\end{abstract}

Palabras claves: Diseño didáctico, reproducibilidad, postítulo de especialización

\section{ABSTRACT}

This article reports on a research focused on analysing the reflections of a working group of teachers who belonged to a brainstorming workshop practice, part of a postgraduate program for professional development, following the Lesson Study methodology. A qualitative approach was used and corresponds to a case study. It was based on a theoretical construct called reproducibility and reflection and conceptualization types of reflection. The findings showed that in discussions between teachers reflections of pedagogical and didactic type emerge naturally and the mathematical discussions are rather scarce. However, when the design of class reflections is installed on the discussion, teachers are focused on reflections of math and didactic type.

Keywords: Instructional design, reproducibility, professional development. 


\section{CONTEXTO}

Un profesor o profesora que participa en un curso o programa de perfeccionamiento para su desarrollo profesional, en ocasiones tiene que realizar diseños didácticos que le permitan poner en acto los conocimientos que va adquiriendo en dicho programa. Es común que tenga que repetir situaciones de aprendizajes en el aula, sin embargo, dicho diseños pueden desnaturalizarse o bien perder su esencia porque en el momento de repetirlo se pueden mutilar o agregar a ello ciertas ideas que provocan un cambio en su propósito didáctico. Frente a lo descrito, desde la didáctica de la matemática, se le denomina a este hecho fenómeno de reproducilidad.

Con el fin de indagar sobre las reflexiones de los profesores cuando tienen que reproducir una misma clase en distintos escenarios (diferentes escuelas y cursos), se realizó un seguimiento a un grupo de trabajo constituido por profesores que realizaban un programa de postítulo de especialización en una universidad chilena. Dichos docentes tenían que realizar una clase basada en resolución de problemas sobre un objeto matemático y usando como referente la Ingeniería Didáctica y aplicar como método el Estudio de Clases Japonés para discutir sobre el diseño y aplicación de la clase en el aula.

\subsection{INGENIERÍA DIDÁCTICA}

La Ingeniería Didáctica (ID) se focaliza en la producción de diseños didácticos para la enseñanza-aprendizaje de un contenido matemático. Una de las características esenciales es que está sustentada en un esquema experimental, basado en las realizaciones didácticas en clase, es decir, sobre la concepción, la realización y el análisis de secuencia de enseñanza (Artigue, 2009). Tiene cuatro fases: la primera es el análisis preliminar que consiste en investigar los antecedentes (epistemológicos, cognitivos y didácticos) que permitirán la construcción de la secuencia didáctica sobre el objeto de estudio; la segunda fase es el análisis a priori, donde se concibe una secuencia didáctica mostrando la organización de ella y sus análisis descriptivo y predictivo; la tercera es de experimentación, donde se prueba la propuesta didáctica o diseño didáctico, se observa en terreno el comportamiento del alumno con respecto a lo planteado y se obtienen las producciones de ellos para el análisis; la cuarta fase es de análisis a posteriori y de evaluación por contraste con la predicción.

El grupo de trabajo de los docentes que participaron en el estudio utilizaron ciertas fases de la ID para realizar su diseño didáctico como: el análisis a priori de las situaciones de enseñanza, lo que permitió predecir lo que ocurriría en la clase, considerando las variables didácticas. Lo anterior posibilita que el docente (al predecir las posibles estrategias de solución de sus estudiantes, los posibles errores, las posibles dificultades) pueda, a posteriori, evaluar la clase en términos de lo que se pensó y de lo que realmente ocurrió en el aula.

\subsection{METOdOLOGÍA DE ESTUDIO DE CLASES}

El jyugyo kenjyu o Estudio de Clases (EC) es un proceso en el cual los profesores desean mejorar progresivamente sus métodos de enseñanza, trabajando con otros profesores para 
examinarse y criticarse mutuamente las técnicas de enseñanza (Isoda, Arcaví y Mena, 2008).

Las características esenciales son el trabajo colaborativo y la reflexión matemática, didáctica y pedagógica que realizan los profesores. Se distinguen tres etapas, las cuales se definen a continuación, tomando como referencia a Isoda et al (2008).

- Fase 1: Preparación de la clase. En esta fase, los profesores en conjunto determina un contenido matemático y diseñan una clase considerando: el currículo, los textos escolares, los materiales didácticos. Es un proceso que se inicia con la búsqueda y selección de recursos o medios relevantes que le permitan establecer el propósito de la clase. Se discute en el grupo de trabajo sobre dicha selección, de tal modo de refinar el diseño de la clase sobre la base de las necesidades efectivas de los alumnos (contexto de la enseñanza). Dados todos estos elementos, se reúnen y se redacta un plan de clase, el cual considera: objetivo de la unidad de aprendizaje, meta de aprendizaje, actividades de aprendizaje (o el tipo de tarea que realizará el alumno), las intervenciones del docente, la distribución del tiempo. Además, en esta parte se hace una predicción de lo que pueda suceder en la clase, en relación a las posibles respuestas de los alumnos, las posibles dificultades y los posibles errores. Es decir, se realiza un análisis predictivo considerando elementos de la didáctica de la matemática.

- Fase 2: Experimentación o aplicación de la clase. Diseñado y validado el plan de clases entre el grupo de trabajo, un profesor realiza la clase. A esta sesión acuden los docentes que conforman el grupo de trabajo con el objetivo de observar la clase y registrar dichas anotaciones, además se filma la clase. Los observadores no intervienen en la clase, sino que pueden pasearse por la sala de clases analizando lo realizado por los alumnos.

- Fase 3: Discusión de la clase. Es una etapa de reflexión matemática, didáctica y pedagógica, sobre la base del plan de clase diseñado en conjunto. Se hacen observaciones sobre la puesta en práctica del diseño. Esta sesión la inicia el profesor que aplicó la clase en su escuela, enseguida los otros profesores opinan, dan ideas y cuestionan decisiones del profesor o bien sobre los recursos que se utilizaron. Es una fase de preguntas que se plantean los docentes y analizan la efectividad de dicha clase en términos precisos del logro de aprendizaje.

Finalizada la discusión de la sesión de clase (Fase 3), se readecua el diseño atendiendo a las discusiones planteadas en pos de mejorar la enseñanza aprendizaje del tema matemático seleccionado. Y se vuelve aplicar la clase en la misma escuela por otro profesor o en otra escuela con otro docente, es un proceso cíclico como se muestra en la figura 1. 


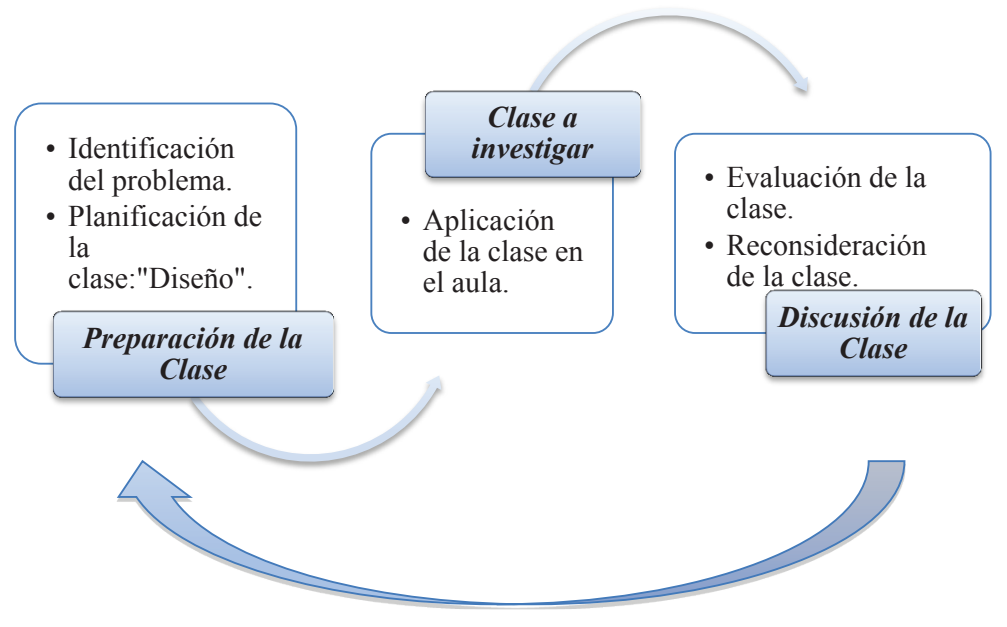

Artigue (2009) expone, en relación a la metodología de Estudio de Clases, que se trata de un dispositivo cuya difusión ha sido acelerada a nivel internacional en la última década y que puede ser vista como una forma de ingeniería didáctica que se calificaría como ID en formación. Agrega que es una respuesta cultural a la pregunta del desarrollo profesional de los profesores en una institución dada. El sistema educativo japonés, sin un control teórico explícito pero debido a la atención que tiene por una serie de evaluaciones y comparaciones, se convirtió en un objeto de estudio para el cual se ha desarrollado un tipo de discurso tecnológico.

De este modo, el Estudio de Clases aporta con un método para realizar los talleres de reflexión de la práctica. En este contexto se decidió investigar sobre el proceso que desarrollaron los profesores en un programa de formación continua para el desarrollo profesional.

\section{ANTECEDENTES}

Distinguir la naturaleza del conocimiento del profesor y sus reflexiones a partir de su propia práctica no es simple, son numerosas las investigaciones que analizan precisamente el conocimiento profesional del profesor y de alguna forma han intentado definirlo. Ponte y Champan (2006) mencionan que el estudio de los profesores y la enseñanza ha sido un campo activo por un largo tiempo en particular en la comunidad del $\mathrm{PME}^{1}$. Señalan que en la década de los ochenta toman gran impulso los estudios focalizados en el profesor, los representantes de esas investigaciones son: Elbaz(1983), quién identificó el conocimiento práctico del profesor y cómo los profesores encapsulan dicho conocimiento; Shulman

PME Psychology of Mathematics Education 
(1986), que expone las ideas sobre el conocimiento pedagógico del contenido (PCK); Schön (1983), que pone atención y promueve la idea sobre la práctica reflexiva, indicando al respecto que cuando se requiere una acción, los profesionales actúan sobre la base de lo que saben sin reparar en lo intelectual o el conocimiento formal de la práctica. Para un profesor reflexionar la práctica tiene que ver con el contenido y los conocimientos pedagógicos relacionados exclusivamente con el contenido.

Azcárate (2004) se refiere al desarrollo profesional y lo vincula a la evolución por parte del docente en la capacidad de reflexión en y sobre la práctica, diagnosticando, comprendiendo para descubrir, criticar y modificar los referentes, esquemas y creencias que subyacen a la misma. Agrega que los profesores son capaces de diseñar, gestionar la puesta en práctica y evaluar propuestas curriculares sin olvidar la complejidad del contexto educativo. También menciona que el conocimiento docente es un conocimiento práctico, es complejo e integrador, es crítico y es profesionalizado sobre la enseñanza de los contenidos. Señala la necesidad de las acciones de formación inicial y continua, y la investigación acerca del fuerte vínculo que deben tener con la práctica docente actual o futura, centrándose preferentemente en procesos de investigación relacionados con algunos de los aspectos específicos implicados con la enseñanza-aprendizaje de las matemáticas.

Con respecto al cómo y qué aprende el profesor en procesos de formación continua, Llinares (2007) señala que el aprendizaje del profesor de matemáticas (tanto en su contexto de formación inicial como en el de formación continua) pasa por llegar a comprender la enseñanza de las matemáticas de una determinada manera, aprender a realizar las tareas, y usar y justificar los instrumentos que la articulan en un contexto institucional.

Perrin-Glorian, Deblois y Robert (2008) remarcan la complejidad de poner en práctica los conocimientos matemáticos que los profesores se apropian. Las autoras revisan la literatura en relación a un estudio que realizan en el contexto del desarrollo profesional docente e indican lo difícil que es organizar la variedad de conceptos a considerar. Identifican tres cuestiones principales. La primera, tiene relación con los cambios de paradigmas propuestos en la formación de profesorado en ejercicio que plantea nuevos problemas en las prácticas de enseñanza. Lo anterior incluye la investigación sobre la formación del profesorado de matemáticas que muestra la importancia de la adaptación, la profundidad y la necesaria articulación de los conocimientos. También, muestran lo difícil que es para los profesores adquirir conocimientos adaptables para gestionar aprendizaje de matemáticas de los estudiantes con actividades desafiantes en el aula. Por lo cual, indican que es importante estudiar la enseñanza en su contexto. La segunda, tiene relación con los cambios que tienen que hacer los profesores por las reformas o nuevos programas de estudio. Señalan que es difícil para los docentes hacer esos cambios y que algunos estudios muestran que cambios aislados no son suficientes para garantizar una mejora real en la práctica. Las dificultades vienen dadas por las imbricaciones entre las creencias y conocimientos de los profesores. La tercera, tiene relación con la importancia de la construcción de conceptos o sistemas capaces de tomar en consideración la variedad de trabajo de los profesores (planificación, análisis, interacciones en el aula, incluyendo las relaciones con los padres, etc.).

Estos antecedentes nos llevan a problematizar e indagar sobre las reflexiones que realizan profesores y profesoras en el marco de un programa de formación continua para articular teoría y práctica. De este modo se plantea, ¿Qué tipo de reflexiones emergen en discusiones de grupos de trabajos de docentes que diseñan y aplican una misma situación de enseñanza en distintos escenarios? 


\section{ENFOQUE TEÓRICO}

La investigación consideró como referente teórico el constructo "reproducibilidad", puesto que los docentes tuvieron que repetir los diseños didácticos en diferentes escuelas. Considerando que para reproducir una misma clase, hay que extrapolar ciertos elementos que permiten el logro didáctico de la propuesta de enseñanza.

También se consideró la conceptualización de reflexión, para identificar los tipos de reflexiones que surgieron en la discusión de los profesores que participaron en el equipo de trabajo.

\subsection{REPRODUCIBILIDAD}

$\mathrm{Al}$ revisar algunas referencias sobre la palabra "reproducibilidad", ésta aparece en varios documentos vinculada estrechamente con el método científico, su uso por tanto es en el área de la ciencia, tiene estrecha relación con volver a "hacer" o "repetir" una experiencia esperando que, a partir de esto, se obtengan resultados similares.

En esta investigación se entendió por "reproducibilidad" a la forma en que una situación de aprendizaje puede ser instalada en distintos escenarios, para extrapolar los elementos que permiten que la situación en sí misma no pierda su esencia, relacionado con el logro del objetivo didáctico. Algunos ejemplos en donde es posible observar la reproducibilidad a nivel de una institución "la escuela", en la cual hay un profesor y alumnos que tienen que aprender en el marco de dicha institución son:

- un profesor que diseña una clase para un grupo determinado de estudiantes y luego tiene que replicar esa clase con otro grupo en la misma institución o en otra diferente;

- $\quad$ un profesor que tiene que replicar la clase o situaciones de enseñanza aprendizaje a lo largo de su ejercicio profesional.

Desde la didáctica de la matemática, el constructo reproducibilidad emerge en la década de los ochenta. Al respecto Brousseau (1986), citado en Artigue (1995), escribe sobre los fenómenos de obsolescencia y relaciona el hecho de que un profesor de un año a otro reproduce condiciones para que sus alumnos tengan los mismos resultados en la comprensión de un concepto. Sin embargo, en lugar de reproducir las condiciones, deja libre las trayectorias y reproduce una "historia" similar a la de años anteriores, pero que desnaturaliza las condiciones didácticas que garantizan una significación correcta de los estudiantes. Es decir, que al repetir situaciones de enseñanza en el aula, pueden desnaturalizarse o bien perder su esencia porque en el momento de repetirlas se pueden mutilar o agregar a ellas ciertas ideas que provocan un cambio en su propósito didáctico. Frente a lo descrito, la reproducibilidad es entendida como un fenómeno didáctico (Brousseau, 1986). Desde esta mirada, entonces, la reproducibilidad en estricto rigor no es posible. Artículos de Arsac (1989), Arsac (1992), Lezama (2005), Artigue (2008) muestran que el profesor es un actor relevante en el momento de reproducir diseños didácticos y hay que considerarlo en forma global, tanto en el conocimiento de las dinámicas de aula como en los fundamentos de las decisiones que realiza. Surge entonces la idea de provocar la discusión y reflexión sobre la reproducibilidad en el grupo de trabajo de los docentes que participaron en la investigación. 
La reflexión sobre el constructo reproducibilidad permitió poner en evidencia que frente a la necesidad de realizar un diseño didáctico para el aprendizaje del teorema de Pitágoras, y ponerlo en escena en distintas escuelas y grupos de alumnos, los docentes debían considerar el fenómeno de la reproducibilidad, con el fin de mantener la esencia o propósito de la situación de aprendizaje.

\subsection{REFLEXIÓN}

La reflexión en el ámbito de la educación, en particular en la formación inicial y continua de profesores, se ha investigado durante décadas. Como consecuencia de dichos estudios, se postula que cualquier programa de formación inicial y continua de profesores ha de considerar la integración del conocimiento científico y del conocimiento práctico. El conocimiento práctico está relacionado estrechamente con el saber hacer del profesor, es decir, es un conocimiento en acción sostenido en la experiencia, reflexión sobre la experiencia y conocimiento teórico (Ponte, 1994), citado por Llinares (2005).

Por otra parte, qué es un profesional reflexivo o qué significa que el docente reflexione sobre su propia práctica no está tan claro en la literatura. Sin embargo, el representante más potente en esta línea es Schön. Esto se puede observar al revisar artículos y libros relacionados con el tema. Flores (2004) confirma que la corriente del profesor reflexivo que ha cobrado realce en la actualidad es precisamente la de Schön $(1983,1992)$, el cual ha remarcado las diferencias que existen entre la racionalidad teórica y la racionalidad práctica que utilizan los profesionales.

Para esta investigación, la definición de reflexión que se adoptó es la de Sánchez (2010), quien dice que es una actividad de tipo cognitiva y la considera un proceso mental por el cual las acciones, creencias, conocimientos o sentimientos son conscientemente consideradas y examinadas.

Dado que la conceptualización planteada sobre la reflexión es del tipo general, se decidió diferenciar los tipos de reflexiones, de tal modo que permita una identificación en la discusión de los docentes. Sánchez (2010), al respecto, distingue dos tipos de reflexiones:

- $\quad$ una reflexión matemática que relaciona la racionalidad matemática misma con lo que se examina conscientemente en la interpretación de conceptos matemáticos.

- una reflexión didáctica, el proceso en el cual el profesor conscientemente considera su propia práctica, sus valores y acciones asociadas con la misma.

Sin embargo, se hizo necesario introducir un tercer tipo de reflexión, denominada reflexión pedagógica, entendida como aquella que es transversal en los procesos educativos, y que tiene relación con las metodologías de enseñanza, planificación de unidades didácticas, planes de clases y los paradigmas de enseñanza. Esta necesidad surge porque en un proceso de estudio o proceso didáctico, hay un profesor que ayuda a otro u otros a estudiar matemática (Chevallard, Bosch y Gascón, 1997).

Con esta tipificación de la reflexión, se analizaron las discusiones que realizaron los profesores y se identificó en qué dimensión -matemática, didáctica o pedagógica- se situaban dichas reflexiones. 


\section{ENFOQUE METODOLÓGICO}

La metodología de análisis es de tipo cualitativa y corresponde a un estudio de casos. Se realizó el seguimiento a un grupo de trabajo conformado por dos profesoras y un profesor que pertenecían a un curso llamado taller de reflexión de la práctica y realizaron un diseño de clase basado en resolución de problemas sobre el teorema de Pitágoras para alumnos y alumnas de 12-13 años. Este seguimiento se plasmó mientras los profesores del grupo participaban en una experiencia de Estudio de Clases, en la que debían reproducir una misma clase en diferentes escuelas y cursos. Es decir, se deseaba observar el cómo los docentes desarrollaban su discusión y reflexión en las diferentes fases del Estudio de Clases y a partir de ahí identificar la naturaleza de las reflexiones.

Para realizar el análisis, se grabaron los talleres en videos; enseguida, se transcribieron a formato de libreto. Se analizaron los libretos a luz del marco referencial identificando los tipos de reflexiones considerando la reproducibilidad de un diseño didáctico.

\section{ANÁLISIS DE TALLERES DE REFLEXIÓN ENMARCADOS EN EL ESTUDIO DE CLASES}

Presentamos, el análisis de la fase uno y dos del Estudio de Clases para situarnos en la metodología. Se expone en forma detallada la discusión de una clase (es decir, posterior a la experiencia de clase) que fue desarrollada por un profesor del grupo de trabajo, puesto que en esta fase se identificaron elementos innovadores, en el hecho de que un grupo de docentes que diseñaron una clase, la observaran y luego discutieran sobre su gestión, para mejorar el diseño en pos del aprendizaje de los alumnos y para reproducirla en otros escenarios.

\section{FASE 1}

En la primera etapa del Estudio de Clase, se realizó la discusión sobre el contenido matemático correspondiente al Teorema de Pitágoras. Esta reflexión surge al plantear la tarea a los docentes de diseñar situaciones de aprendizajes para estudiantes de 12-13 años. Se inició la discusión, a partir de la pregunta ¿Qué es para ustedes el teorema de Pitágoras?

En las respuestas dadas, se revela que los profesores reconocen el teorema de Pitágoras como una técnica de carácter operacional para encontrar la longitud de los catetos o la hipotenusa en un triángulo rectángulo. El hecho de decir técnica se relaciona con que los docentes no se refieren al teorema en sí, sino que de inmediato surgen dos palabras claves asociadas al objeto matemático: fórmula y medida.

Además, distinguen la fórmula y escriben (en un comienzo de la discusión) $h=a^{2}+b^{2}$, donde $h$ representa la longitud de la hipotenusa y a, b corresponde a las longitudes de los catetos. Posteriormente, corrigen dicha fórmula y escriben $h^{2}=a^{2}+b^{2}$; también identifican que para dicha fórmula hay una condición. Es decir, $h^{2}=a^{2}+b^{2}$, en un triángulo rectángulo, siendo $a, b$ las longitudes de los catetos y $h$ la longitud de la hipotenusa. Se detecta que no hay alusión a la hipótesis y tesis del teorema.

También, visualizan el teorema de Pitágoras mediante la figura 2. Hecho que permite determinar que tienen la noción del teorema a través de registro algebraico y visual. 
Fig. 2: Representación del teorema de Pitágoras dadas por los docentes

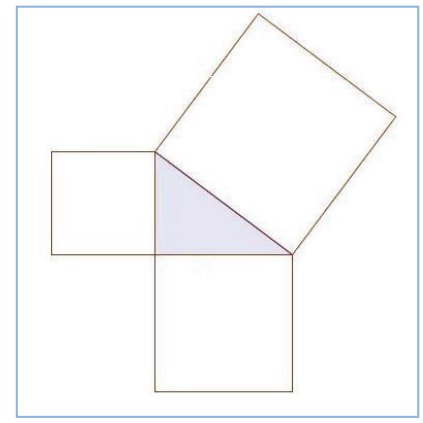

La justificación del teorema de Pitágoras la realizaron en forma pragmática, relacionando la fórmula expresada con las áreas de los cuadrados que se trazan sobre los catetos y la hipotenusa.

En esta fase del Estudio de Clases, los profesores reflexionaron sobre el diseño de la clase, que consistía en un conjunto de actividades. Al inicio de la clase, planteaban una pregunta relacionada con la geometría ¿Cómo se utiliza la geometría en la vida diaria?, enseguida muestran actividades en donde los alumnos tienen que calcular áreas y perímetros de figuras geométricas (cuadrados, rectángulos). En el desarrollo de la clase, plantearon actividades medulares como: calcular el perímetro de un triángulo rectángulo en donde sólo se conocen las medidas de los catetos; el desafío para el alumno fue determinar la longitud de la hipotenusa para calcular el perímetro de la figura; la otra actividad es la verificación del teorema de Pitágoras en forma pragmática.

Los profesores en esta etapa discutieron sobre las actividades diseñadas, se anticiparon a las respuestas de sus alumnos, es decir, buscaban controlar ciertas variables didácticas del diseño de la clase. Lo que se observó, por ejemplo, en el siguiente episodio:

Isidora: Yo creo que al menos en mi clase, no me van a decir que es el teorema de Pitágoras, pero pueden llegar a 5 , por último

Martín: Por la medición de regla

Académica: Entonces, cuando te digan es 5, ¿Qué van a hacer ustedes ahí?

Isidora: No, ahí nos vamos (Risas), yo creo que yo renuncio

Pamela: Yo creo que si van a decir 5, hay que verificar y comprobar porque es 5

En este diálogo los docentes se refieren a una de las actividades medulares de la clase y las posibles respuestas de sus alumnos, están focalizados en una reflexión didáctica.

\section{FASE 2: EXPERIMENTACIÓN DE LA CLASE}

En esta fase, el diseño didáctico (la clase) fue aplicada por cada uno de los docentes, que pertenecen al grupo de trabajo, en cada una de las escuelas en donde trabajaban. Además, se invitó a una cuarta profesora al grupo para que observara las clases y participará en las discusiones de ellas; esta docente no participó en el diseño. En esta etapa, se ubica una idea 
fundamental de la investigación que es el fenómeno de reproducibilidad de las situaciones de aprendizajes. El método que se siguió para esta experimentación fue: un profesor del grupo realizó la clase en su escuela, los profesores restantes observaron la clase, enseguida se discutió y reflexionó sobre la clase aportando ideas para readecuarla en pos del logro didáctico. Posteriormente otra profesora aplica la clase, el resto de los docentes la observa, enseguida se discute sobre su aplicación, y así sucesivamente. Se observaron cuatro clases; en la figura 3 se muestra el proceso descrito.

Fig. 3: Proceso de aplicación y discusión de clases

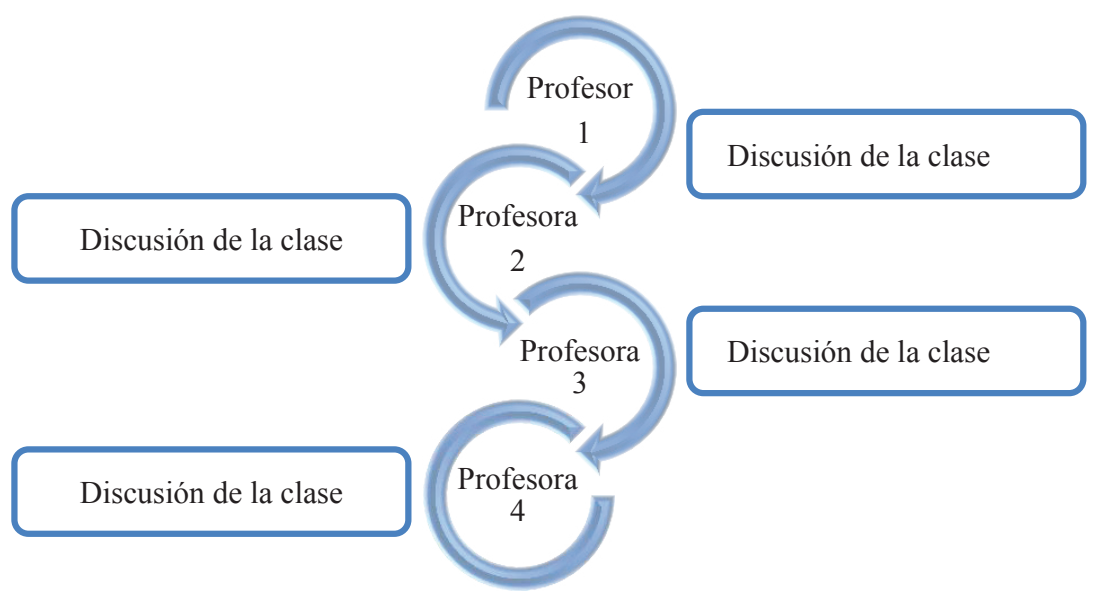

\section{FASE 3: DISCUSIÓN Y REFLEXIÓN DE LA CLASE}

Análisis del taller de discusión de la Clase 1

Se detecta en esta reflexión y discusión sobre la clase del profesor 1 (Martín) varias ideas que se pueden identificar como reflexiones de tipo matemática, didáctica y pedagógica, de acuerdo al marco referencial.

Las reflexiones pedagógicas tienen su foco en la estructura de la clase: inicio, desarrollo y cierre. Los cuatro docentes que participan en la discusión hacen intervenciones en donde es posible detectar las ideas en relación a los tiempos que el profesor ocupa para la clase. El profesor Martín, quién realizó la primera clase, expone sobre la relación del tiempo y los conocimientos previos de sus alumnos, da una visión global de su clase mostrando la estructura de la clase, las docentes que observaron su clase dan su opinión al respecto:

Episodio 1: [ primeras opiniones de la clase del profesor Martín]

Martín: Bien, la clase fue un poco difícil, difícil llevar la clase porque yo pensaba que los niños se acordaban bien de área y perímetro y pensé que iba a ser natural el relacionar esas dos 
medidas, y me di cuenta de que no, a pesar de que se había repasado no fue tan natural como lo pensé, entonces ocupé más tiempo del que tenía presupuestado para eso, entonces me compliqué un poco y después me sentí un poco presionado por los tiempos, no sabía si apurar, eso me cortó un poco. Bueno en el desarrollo de la clase considero que fue (asienta con la cabeza), la mayoría participaba, habían algunos que no participa, pero la mayoría sí participaba, y llegar a lo que se pretendía, por lo menos en un principio.

Isidora: la clase bueno, en sí lo que nosotros diseñamos hay que arreglar algunos detalles y acomodar algo para que se pueda alcanzar el cierre y ver si los niños internalizaron realmente el teorema de Pitágoras o ver si se basó más en los conocimientos de área y perímetro. Bueno, yo encuentro que la clase se dio como la habíamos planificado, la actividad de inicio debió haber sido más rápida, más dinámica y no demorar tanto, quizás como dice Martín el tema de que quería afianzar más el tema de área y perímetro, pero siento que se dio más énfasis del necesario al inicio, para poder abordar más la base que era el desarrollo y ver si aprendieron en el cierre, que por tiempo no se alcanzó a completar.

Pamela: yo al igual que la Isidora concuerdo en que el inicio fue un poquito largo, la clase era del teorema de Pitágoras y yo le hubiese dado un poquito más de énfasis al teorema de Pitágoras. O sea estamos claro que a lo mejor los niños no se acordaban de perímetro y área en sí, pero a lo mejor nosotros lo hubiésemos puesto como un recordatorio y en base a eso empezar con el teorema de Pitágoras.

Romina: [profesora que no participó en el diseño de la clase] ... a ver, la clase me gustó, encuentro que si bien es cierto la clase estaba bien estructurada con sus distintos momentos inicio, desarrollo y su final, siento que faltó un poquito en el cierre haber finalizado, quizás por tiempo, por los mismos nervios no sé o donde habíamos tantos ahí quizás los niños se sentían un poquito más cohibidos porque quizás faltó un poquito más de participación de los alumnos, participar más. Entonces en el cierre creo que me faltó esa parte en la que yo pudiera comprobar el teorema, la comprobación, a través de esta actividad que tenían que recordar y que quedó un poco inconclusa porque faltó tiempo ¿cierto?. Creo que se podría hacer esa modificación acotar un poco al inicio, centrarlo más en lo que era el área del triángulo o específicamente en el del triángulo rectángulo, y desde ahí trabajar más en el desarrollo. Bueno y faltó cerrar bien, hacer realmente la comprobación del teorema.

\section{Análisis Episodio 1}

Las primeras intervenciones del grupo de trabajo y discusión son globales y miran la clase en términos de la estructura que ellos han ideado. Detectan que el objetivo de aprendizaje que era la comprensión del teorema de Pitágoras no se logra percibir en los estudiantes, puesto que el momento de inicio fue extenso por lo cual el momento de desarrollo no se alcanza en su totalidad.

A luz de lo que discuten y opinan en relación a la gestión de la clase que realizó el profesor, se sitúa la reflexión en la dimensión pedagógica, puesto que hacen alusión a la estructura de la clase (inicio, desarrollo y cierre) y los tiempos que se dieron para cada una de esas etapas, esto significa que es una reflexión pedagógica. Por otra parte, hay una discusión que se focaliza en la dimensión didáctica, porque analizan la actividad en términos de gestión lo que conlleva a que el desarrollo de la clase no permite lograr la comprensión del teorema de Pitágoras. También hay esbozo de reflexionar sobre la participación de los alumnos. 
Episodio 2: [el contenido matemático]

En este episodio se observa una de las ocasiones en que los docentes reflexionan en relación al objeto matemático en cuestión.

Isidora: yo siento que hay una corrección que no se hizo y a lo mejor Martín no la alcanzó a ver, pero para mí ese es el teorema y ahí está el teorema y no hay otro.

Pamela: jah! claro, te faltó como la definición del teorema, que la hipotenusa, que el cateto al cuadrado con la hipotenusa, la fórmula, la fórmula en sí.

Isidora: el a más el $b^{2}$ es igual al $c^{2}$

Pamela: Claro, eso como que también faltó la formulita del teorema de Pitágoras.

Romina: Encontré súper bueno esta parte donde dice verifica la hipótesis y la tesis del teorema, porque generalmente uno no le da mucho importancia a lo que son las definiciones o los axiomas, entonces generalmente uno se queda como en el cálculo o en la fórmula, entonces esta parte siento que fue buena porque así los niños pudieron identificar que tenían que comprobar algo, comprobar el teorema que digamos se estaba trabajando, y algunos niños me acuerdo que nombraron que la hipótesis era algo que tenían que cumplir, que tenía que comprobarse, tú la relacionaste con un método científico en ciencias naturales, ahí está esa parte (indicando una hoja), sino el inicio acotarlo un poco más.

\section{Análisis del Episodio 2}

En este episodio, la profesora Romina hace alusión al contenido matemático, señala que el profesor Martín muestra que el teorema está constituido por una hipótesis y la tesis, dado el contexto interpretamos este hecho como una reflexión matemática. Se observa, además, que es una de las ocasiones en que los docentes reflexionan en relación al objeto matemático en cuestión.

Otra interpretación, que se extrae de este episodio, es que no problematizan la matemática o mejor dicho no profundizan en el contenido matemático ya que cuando abordan el contenido en particular se refieren a la escritura o al lenguaje que utiliza el profesor que realizó la clase. Se refieren al teorema de Pitágoras como una definición o bien como la fórmula ausente de significación.

Episodio 3: [gestión de la clase]

Romina: ... se comprendió bien en el momento que tú pusiste los cuadraditos en el área, yo creo que estaba súper bien explicado, quizás lo que faltó un poquito fue que tú diste mucha.

Isidora: ¿información?

Romina: mucha información, quizás ellos [se refiere a los alumnos] tendrían que haber entregado mayor información, y tú no darle tantas pistas para que te dieran información. Pero en general, a mí me gustó bastante la clase.

\section{Análisis episodio 3}

La observación que realizan las profesoras están enfocadas al tiempo y la gestión del profesor, se refieren a las informaciones que el docente entrega a los alumnos, reconocen 
que aportó demasiada información y que hay poca participación de los alumnos en la clase. Dado que, mencionan hechos con participación y gestión ente docente y alumnos, situamos la naturaleza de esta reflexión en la dimensión didáctica, por lo cual corresponde a una reflexión didáctica.

Episodio 4: [actividades de enseñanza y aprendizaje]

Isidora: yo pienso que la actividad número 3, bueno la última la de recortar es una actividad muy buena para que ellos puedan comprobar y clarificar, pero al minuto de entregar el material los niños no sabían qué hacer, no estaba la instrucción clara, porque todavía no tenían bien claro ellos, y eso lo discutimos mucho con el grupo porque estamos con la idea de que el niño descubra.

Romina: yo creo que esta actividad de cierre debiese haber sido parte del desarrollo. Porque con esta tu compruebas el teorema y quizás dándoles más tiempo a los niños porque aquí como que les costó ¿qué hago con esto? ¿No es cierto? Entonces las instrucciones no estaban muy claras, entonces en ese tiempo él quizás hubiese descubierto si le hubiera alcanzado el tiempo, si ponemos esta como parte del desarrollo, ahi el alumno tiene que probar y buscar distintos caminos, porque si bien es cierto estaban las líneas digamos donde tenía que cortar, a lo mejor si no hubiesen estado las líneas.

\section{Análisis del episodio 4}

En este episodio, las docentes sitúan sus discusiones en una de las actividades medulares de la clase, dicha actividad tenía por objetivo que los alumnos comprobarán en forma pragmática el teorema de Pitágoras. Las profesores reconocen que es una buena actividad porque los alumnos pueden comprobar y clarificar, sin embargo, se dan cuenta que las instrucciones para resolver la actividad no estaba lo suficiente clara. Además, una de las docentes plantea que la actividad debiera ser parte de desarrollo de la clase. Las reflexiones de las profesoras se sitúan en una dimensión didáctica, porque hicieron referencia a la claridad de la tarea matemática que se propuso y por otra parte una de ellas propone cambiar dicha tarea al desarrollo de la clase. En este caso, se distingue que la naturaleza de la discusión es una reflexión didáctica.

En el episodio 5 se muestra las interacciones que tienen los docentes en el cuestionamiento de otra de las situaciones medulares de la clase, que corresponde a la situación 3 (figura 4). 
Fig. 4: Situación 3 planteada a los alumnos

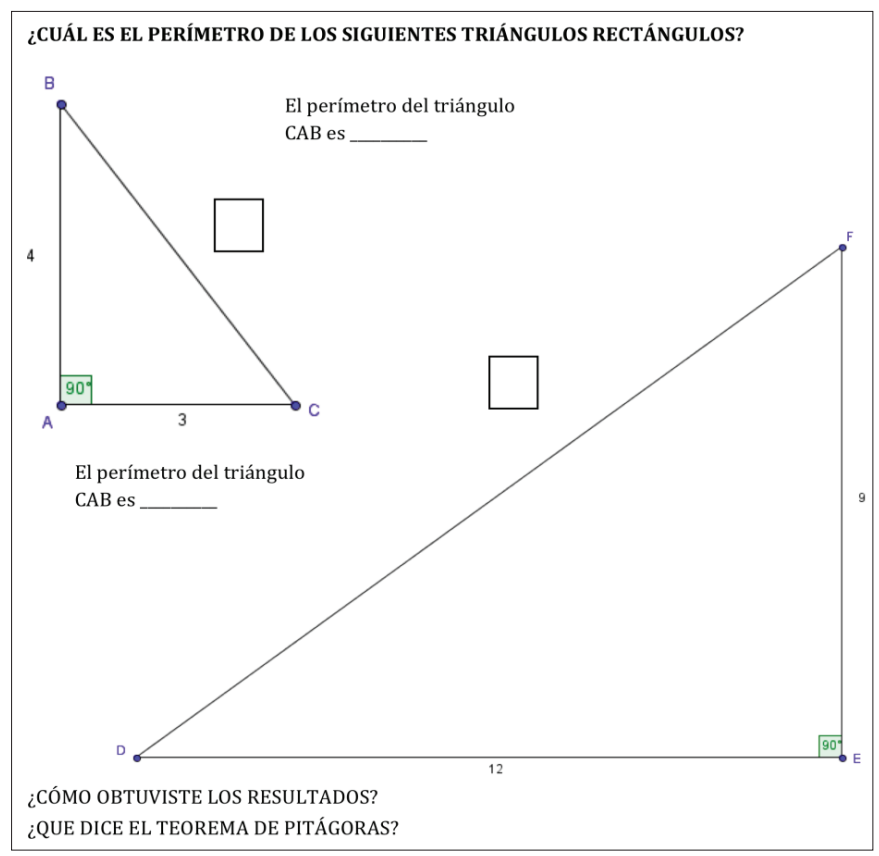

Episodio 5: [actividades de enseñanza y aprendizaje]

Isidora: [se refiere a la situación 3] El primer triángulo da justo 3,4 y 7, y con la regla daba 7, entonces yo pienso que eso llevo a los niños a pensar que había que sumar 3 y 4 lo que daba 7. Isidora: Pero se contradice con el segundo porque los niños midieron, yo estaba mirando al niño de al lado, y no daba 12 daba como 14,5. Pero el primero llevaba al niño a ese error, o sea a esa concepción. No sé si se fijaron pero 10 de los niños dijeron lo mismo y 12 de los niños asumieron de la otra manera.

Martín: Pero, ¿cuál es el objetivo real de esta actividad?

Isidora: Que ellos tuvieran la duda, que ellos se presentaran ante una dificultad, ¿cómo saco el perímetro si me falta un lado? ¿Cómo consigo el lado faltante?

\section{Análisis del episodio 5}

La profesora Isidora, en su primera intervención pone en evidencia que los niños se equivocaron al responder que la hipotenusa es 7 , puesto que al comprobar con la regla efectivamente en la figura, la hipotenusa del triángulo BAC media $7 \mathrm{~cm}$. Por lo cual, interpreta este error de los niños como consecuencia del dibujo. También, la docente señala que con el segundo triángulo, los niños pensaron que al sumar 12 y 9 obtenían la longitud de la hipotenusa. Sin embargo, al comprobar y medir, se obtenía 14,5. La naturaleza de esta reflexión es de tipo didáctica, frente al error de los alumnos, cuestionaron el dibujo. Es decir, la profesora trata de comprender por qué el alumno se equivocó y da sus argumentos. 
Por otra parte, el profesor Martín frente a esta intervención se sitúa desde la dimensión pedagógica, puesto que hace alusión al objetivo de la actividad. Trata de conectar la acción de los niños con el objetivo de la situación.

Mientras que la profesora Isidora responde en el sentido que el propósito de la actividad es llevar a los niños a una situación que les crea duda, inestabilidad y que requiere una respuesta matemática, es decir su reflexión es de tipo didáctica.

Cuestionan por una parte el dibujo de los triángulos, la medida de los catetos era 3 y 4, varios alumnos dijeron de inmediato que la hipotenusa media 7. Dos ideas se discuten: cuáles son los errores de los alumnos y el objetivo de la actividad.

\section{Comentario del episodio 5}

Los docentes reconocen que la situación 3 (figura 4) es un problema, pero a su vez cuestionan esta actividad. Sin embargo, no llegan a consenso para realizar la modificación.

Proponen modificar dichas actividades en el sentido de reducir el tiempo para que los alumnos respondan, en otras palabras, no cambian el tipo de actividad sino la gestión de ella.

Después del episodio 5, y dado que varios alumnos cometen un error en la situación 3 y han analizado el dibujo que propusieron, los profesores discuten sobre cambiar o no las medidas del triángulo en el dibujo para poder aplicar en otros escenarios esta situación. Pues, el tener las medidas de los catetos, la única posibilidad para los alumnos es medir con regla porque no tienen como conocimiento el teorema de Pitágoras. Así, la posibilidad de cambiar la actividad cuando sea aplicada en otro curso es fundamentada en la acción que realizan los alumnos y teniendo en vista el objetivo de plantear esta situación de aprendizaje.

En los episodios 6 y 7 se muestra cómo los docentes reflexionan sobre la reproducibilidad del diseño de clase, puesto que el profesor Martín fue el primero en aplicar las situaciones de aprendizaje. Posterior a este taller, las profesoras Isidora, Pamela y Romina aplicarían las mismas situaciones en sus respectivos cursos.

\section{Episodio 6}

Isidora: Yo le contaba a Romina podemos tener el plan exactamente igual pero la forma es de cada uno, la impronta es muy distinta, entonces yo le decía a las chiquillas que tiendo a hablar muy rápido y ser muy ágil, entonces a mí me sobra tiempo en las clases, entonces yo sé que el inicio lo voy a hacer más rápido y me va a alcanzar el tiempo, entonces en el desarrollo voy a dar énfasis en que tratemos de entender más para que al final puedan verificar, y a lo mejor si resulta, pero también les voy a dar la opción de que lo corten como ellos quieran.

A: Pero ahí ya modificaste la actividad.

Romina: Es que yo creo que Martín no alcanzó porque hubo tiempos perdidos, como por ejemplo la introducción, no estoy cuestionando eso, pero esos son tiempos perdidos. Porque estaba planificada para un tiempo la actividad.

Isidora: Hay que reforzar el desarrollo para que tenga éxito la modificación.

A: ¿Y qué más?

Isidora: Martín lo hizo en función del tiempo, quedaban 15 minutos y teníamos que pasar esta actividad, entonces ya no podía, pero yo pienso que la idea de eso es ¿Qué entendieron? ¿Qué es lo que ellos realmente lograron comprender del teorema de Pitágoras? O sea que podemos 
llegar al valor de la hipotenusa, que los valores son los que sumados dan el otro, entonces comprobemos esa verdad, como dice Martín pongámoslo en duda. ¿Haber se logra o no? Pero todavía no era apropiado lo que era.

Pamela: Yo creo que hay que acortar el inicio para poder hacer esa actividad

\section{Análisis del Episodio 6}

La reflexión que realiza el grupo de trabajo para reproducir la misma clase se focaliza en dos dimensiones; la pedagógica y la didáctica.

Las profesoras se refieren al tiempo que utilizó el profesor en la gestión de las actividades, mencionan una readecuación de los tiempos para el desarrollo de las situaciones, esta es una reflexión de tipo pedagógica.

Por otra parte, la profesora Isidora reflexiona en relación a la actividad presentada y se focaliza en la comprensión del contenido matemático por parte de los alumnos, ella señala que la idea de la situación es si los alumnos logran la comprensión del teorema de Pitágoras. La naturaleza de esta reflexión es didáctica, puesto que se centra en la actividad y el logro didáctico.

En el siguiente episodio, se expone las interacciones de los docentes frente a la pregunta que hace la académica.

\section{Episodio 7}

A: Hay tres profesores que tienen que hacer la misma clase y la idea no es cambiar la actividad ¿Qué tenemos que cambiar? ¿En qué tenemos que fijarnos?

Martín: En el tiempo.

Pamela: Faltó el tiempo uno de ellos.

Isidora: Énfasis en el desarrollo.

Romina: Énfasis en la actividad de problemas que ese es problema.

Isidora: Ese es el desarrollo.

Pamela: Y acortar el tiempo de inicio, o sea la actividad de inicio.

A: De otra manera ¿qué no podemos borrar?

Pamela: La actividad central.

A: ¿Cuál?

Pamela: Esta [apuntando una hoja]

Pamela: No sé si no se podrá borrar, pero yo creo que aquí podemos plantearlo como la hipotenusa.

Romina: Se puede cambiar la pregunta del profesor.

Isidora: Qué comprendan, conocer y comprender el teorema de Pitágoras. ¿Y eso que significa entonces? Dar un significado. Hay varias cosas que a partir de la discusión los profesores que van a realizar, ya tienen más elementos para darse cuenta.

\section{Análisis del episodio 7}

En las interacciones de los docentes frente al planteamiento de repetir la misma clase, considerando no cambiar la actividad, las profesoras se acercan a la dimensión matemática. Ellas señalan, que deben colocar énfasis en el desarrollo de la clase y frente a la pregunta 
¿qué tienen que cambiar? señalan el tiempo y en relación a la pregunta ¿en qué debemos fijarnos? responden en relación al logro didáctico, en este caso que comprendan el teorema de Pitágoras. A partir de esto, el análisis de las docentes se sitúa en el contenido matemático que los alumnos tienen que aprender, es decir, se puede interpretar que para repetir la clase hay que considerar el aprendizaje esperado o logro didáctico. Por tanto, es una reflexión de tipo matemática.

En resumen, el análisis del grupo de trabajo de los docentes, después que el profesor realizó la clase, conduce a develar tipos de reflexiones, en el escenario del Estudio de Clases antes el interés de repetir la misma clase en distintas escuelas.

Además, en la trayectoria de reflexión y discusión se observa que las reflexiones didácticas y pedagógicas son más abundantes y en ellas los docentes exponen sus ideas sobre: las situaciones de aprendizajes, el constructo reproducibilidad, las respuestas de los alumnos, los errores que detectaron cuando los alumnos trabajaron las actividades, los tiempos que se dieron para que los alumnos accionaran en las tareas, las veces que el profesor dio pistas para que los estudiantes llegaran a la respuesta.

Las reflexiones de tipo matemática son escasas, aunque frente a la consideración de aplicar las situaciones de aprendizaje en otros cursos y escuelas (reproducir la misma clase), se detecta que se aproximan con mayor énfasis a la dimensión matemática, puesto que su reflexión radica en el contenido o significado del teorema de Pitágoras.

\section{CONCLUSIÓN Y PROYECCIONES}

El análisis de esta experiencia nos permite concluir que la metodología de Estudio de Clases, instalada como método de formación continua para el desarrollo profesional, entregó un escenario para crear ambientes de discusión y reflexión sobre la práctica de los profesores. Esto quedó en evidencia en las discusiones posteriores a la aplicación de la clase en sus dimensiones matemática, didáctica y pedagógica.

A la pregunta ¿qué tipo de reflexiones emergen en discusiones de grupos de trabajos de docentes que diseñan y aplican una misma situación de enseñanza en distintos escenarios? Podemos señalar que hay dos tipos de reflexiones que emergen en forma más natural por parte de los profesores; pedagógicas y didácticas, siendo la de naturaleza matemática inducida por la necesidad de estabilizar o reproducir el propósito didáctico que se propusieron en su diseño.

La proyección de este estudio es realizar indagaciones sobre los aprendizajes logrados por los estudiantes, a partir, de la reflexión que realizan los docentes sobre una misma clase aplicada en distintos escenarios, al amparo de la metodología Estudio de Clases. Es decir, estudiar el impacto del Estudio de Clases en los logros de aprendizaje de los estudiantes.

\section{REFERENCIAS BIBLIOGRÁFICAS}

Arsac, G. (1989). Le rôle de professeur - aspects pratiques et théoriques, reproductibilité. Cahiers du Séminaire de Didáctique des Mathématiques et de l'informatique. Grenoble, France: IMAG-LSD. Arsac G., Balachef N., Mante M. (1992) Teacher's Role and reproducibility of didactical situations. Educational Studies in Mathematics, 23 (1), 5-29. 
Artigue, M. (1995). Ingeniería didáctica. En P. Gómez (Eds.), Ingeniería didáctica en educación matemática. Un esquema para la investigación la innovación en la enseñanza y el aprendizaje de las matemáticas (pp. 33-59). México: Grupo Editorial Iberoamérica.

Artigue, M. (2008).Didactical Design in Mathematics Education. En C. Winslow (Ed.), Nordic Research in Mathematics Education (pp. 7-16). Copenhagen: Proceedings from NORMA08.

Artigue, M. (2009). L'Ingénierie didactique: Un essai de Synthèse. En C. Margolinas, M. AbboudBlanchard, L. Bueno-Ravel, N. Douek, A. Fluckiger, P. Gibel y F. Vandebrouck (Eds.). En Amont et en aval des Ingénieries Didactiques (Vol. 1, pp 225-237), Clermont -Ferrand: XV 15 École d'été de didactique des mathématiques.

Azcárate, P. (2004). Los procesos de formación: En busca de estrategias y recursos. Seminario de Investigación presentado en VIII Simposio ( SEIEM). Recuperado de http://www.seiem.es/publicaciones/archivospublicaciones/actas/Actas08SEIEM/VIIISimposio.pdf.

Bosch, M., Chevallard, Y., Gascón J. (1997). Estudiar Matemáticas: El eslabón perdido entre la enseñanza y el aprendizaje. Barcelona: ICE-HORSORI.

Brousseau, G. (1986). Fondements et méthodes de la didactique des mathématiques. Recherche en Didactique des Mathématiques, 7 (2), 33-112.

Elbaz, F. (1983). Teacher thinking: A study of practical knowledge. London: Croom Helm.

Flores, P. (2004, septiembre). Profesores de matemáticas reflexivos: formación y cuestiones de reflexión. Ponencia presentado en VIII Simposio ( SEIEM)

Recuperado de http://www.seiem.es/publicaciones/archivospublicaciones/actas /Actas08SEIEM/Flores_ponencia.pdf

Isoda M., Arcavi A. y Mena A. (2008). El Estudio de Clases Japonés en Matemáticas, Chile. Valparaíso: Ediciones Universitarias de Valparaíso, Pontificia Universidad Católica de Valparaíso.

Lezama J. (2005). Una mirada socioepistemológica al fenómeno de reproducibilidad, Revista Latinoamericana de Investigación en Matemática Educativa, 8, 339-362.

Llinares, S. (2005, julio). Relación entre teorías sobre el aprendizaje del profesor de matemáticas $y$ diseño de entornos de aprendizajes. Conferencia invitada presentada en el Congreso Iberoamericano de Educación Matemática (V CIBEM), Oporto, Portugal.

Llinares S. (2007). Formación de profesores de matemáticas. Desarrollando entornos de aprendizajes para relacionar la formación inicial y el desarrollo profesional. Conferencia invitada en la XIII Jornadas de Aprendizaje y Enseñanza de las Matemáticas, JAEM, Granada. Recuperado de http://rua.ua.es/dspace/bitstream/10045/853/1/llinares-jaem-granada07.pdf

Perrin-Glorian, M. J., Deblois, L. y Robert, A. (2008). Individual Practising Mathematics Teachers. En K. Krainer y T. Wood (Eds.) Participants in Mathematics Teacher Education (Vol. 3 pp. $35-$ 59). The International Handbook of Mathematics Teacher Education.

Ponte, J. (1994) Mathematics teachers' professional knowledge. En J.P. Ponte \& J.F. Matos (Eds), Proceedings of the 18th PME International Conference, 1, 195-210.

Ponte J. y Chapman (2006). Mathematics teachers' knowledge and practices. En A. Gutierrez \& P. Boero (Eds.). Handbook of research on the psychology of mathematics education: Past, present and future (pp. 461-494). Roterdham: Sense. Recuperado de http://math.unipa.it/ grim/YESS-5/06\%20Ponte-Chapman-PME\%20Handbook.pdf.

Sánchez, M. (2010). How to stimulate rich interactions and reflections in online mathematics teacher education? (Tesis de Doctorado no publicada), IMFUFA tekst nr. 472 - 2010. Universidad de Roskilde: Dinamarca. Recuperada de: http://milne.ruc.dk/imfufatekster/pdf/472.pdf.

Schön, D. (1983). The reflective practitioner: How professionals think in action. Avebury: Aldershot Hants.

Shulman, L.S. (1986). Those who understand: Knowledge growth in teaching. Educational Researcher, 15 (2), 4-14. doi: 10.3102/0013189X0/5002004 